\title{
THE EFFECT OF AN AXIAL MAGNETIC FIELD ON THE SPONTANEOUS EMISSION FROM AN ARGON HOLLOW CATHODE DISCHARGE
}

\author{
J.J. ROCCA, G.J. FETZER and G.J. COLLINS \\ Department of Electrical Engineering, Colorado State University, Fort Collins, CO 80523, USA
}

Received 16 December 1980

Revised manuscript received 27 April 1981

An applied longitudinal magnetic field is observed to decrease the equilibrium population of excited argon ion levels in a hollow cathode discharge, whereas the excited state populations of neutral argon atoms are not significantly altered. The longitudinal magnetic field is shown to decrease the effective density of beam electrons in the negative glow.

Hollow cathode discharges (HCD) have a high-energy component in the electron energy distribution which makes them an attractive medium for ion lasers [1]. Schuebel [2], for example, reported cw argon ion laser oscillation in a HCD. Moreover, the use of an axial magnetic field in a conventional positive column argon ion laser is known to significantly increase the laser power output [3]. The effect of both axial and transverse magnetic fields in metal vapor-noble gas hollow cathode discharges have been studied in the past [4-7] but, to our knowledge, no studies of pure argon discharges have been reported.

We have examined the effects of an axial magnetic field in an argon HCD by making measurements of the spontaneous emission intensity from a variety of neutral and ionized levels. High-purity graphite was selected as cathode material because of its low sputtering yield. The graphite cathode employed was $13 \mathrm{~cm}$ long and had a $4 \mathrm{~mm}$ inner diameter cylindrical cavity connected to the anode region by a $1.5 \mathrm{~mm}$ wide slot. A stainless steel rod $2 \mathrm{~mm}$ in diameter was used as the anode. Both electrodes were placed inside a water cooled quartz vacuum envelope so that the axis of the hollow cathode was approximately coaxial with the quartz tube. The electric discharge was in a direction perpendicular to the optical axis. The vacuum envelope was surrounded by a $27 \mathrm{~cm}$ long solenoid capable of providing a magnetic field parallel to the optical axis with a field up to $800 \mathrm{G}$.
Line intensities of selected spectral lines of $\mathrm{Ar} I$ and Ar II have been measured using a $1 / 2 \mathrm{~m}$ Jarrell Ash spectrometer and a 31034 RCA photomultiplier. The line intensities have been directly plotted as a function of the magnetic field strength with an $X-Y$ chart recorder. The light emitted from the cylindrical cathode discharge was collimated in order to eliminate light collection from the small positive column region. In the case of spontaneous emission measurements done at constant current the discharge power supply was operated in its current mode and the discharge voltage varied.

The intensity of the spontaneous emission in the Ar II $3 p^{4} 4 p \rightarrow 3 p^{4} 4 s$ transitions listed below have been measured as a function of the magnetic field with the current density as parameter: $4879.8 \AA\left({ }^{2} \mathrm{D}_{5 / 2}-{ }^{2} \mathrm{P}_{3 / 2}\right)$, $4764.8 \AA\left({ }^{2} \mathrm{P}_{3 / 2}-{ }^{2} \mathrm{P}_{1 / 2}\right), 5145.3 \AA\left({ }^{4} \mathrm{D}_{5 / 2}-2 \mathrm{P}_{3 / 2}\right)$, $4965.0 \AA\left({ }^{2} \mathrm{D}_{3 / 2}-{ }^{2} \mathrm{P}_{1 / 2}\right)$ and $4579.3 \AA\left(2 \mathrm{~S}_{1 / 2}-{ }^{2} \mathrm{P}_{1 / 2}\right)$. The data were taken at $2.5,2,1.5$ and 1 Torr. All observed ion transitions displayed the same qualitative behavior; that is, a significant decrease of the spontaneous emission intensity is observed with increasing magnetic field, as shown in fig. 1 for the representative $5145.3 \AA$ line. At $800 \mathrm{G}$ the intensity of the Ar II emission drops as much as $50 \%$ from the intensity level at zero applied field. Current densities (total currend divided by cathode area) up to $80 \mathrm{~mA} / \mathrm{cm}^{2}$ have been used in our experiments.

The Ar I behavior was in marked contrast to that 


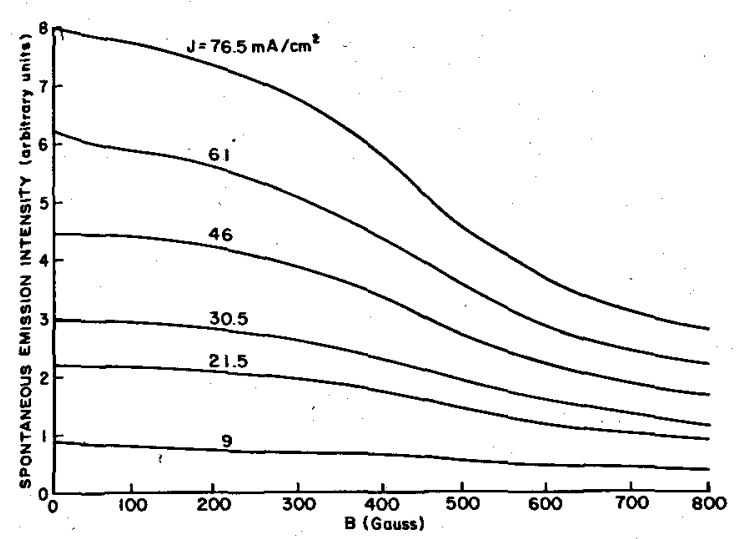

Fig. 1. Spontaneous emission intensity of Ar II $5145.3 \AA$ line as a function of magnetic field strength. Filling pressure 2 Torr of argon.

observed for Ar II. The variation of the emission intensity for the selected neutral argon transitions was rather insensitive to the magnetic field intensities, as shown in fig. 2 for the representative $5916.6 \AA$ $\left(5 d[3 / 2]_{1}-4 p[5 / 2]_{2}\right)$ transition of Ar I. In fact we also monitored the $4198.3 \AA\left(5 \mathrm{p}[1 / 2]_{0}-4 \mathrm{~s}[3 / 2]_{1}\right)$, $4200.7 \AA\left(5 \mathrm{p}[5 / 2]_{3}-4 \mathrm{~s}[3 / 2]_{2}\right)$ and $4510.7 \AA$ $\left(5 \mathrm{p}[1 / 2]_{0}-4^{\prime} \mathrm{s}[1 / 2]_{1}\right)$ emission (the latter as a check on the population of the $5 \mathrm{p}[1 / 2]$ level). A few of the atomic lines did show a small reduction in intensity with increasing magnetic field, but this change was always less than $20 \%$ of their value at zero field.

The spontaneous emission intensity of the Ar II spectral lines was measured to increase linearly with discharge current, for all magnetic field values (fig. 3 ). In sharp contrast, all of the neutral lines examined display a clear saturation with increasing current (fig. 3). The linear increase of the Ar II intensities with increasing current density arises, as pointed out by Schuebel, because single step excitation involving beam electrons and ground state neutral argon fully accounts for the majority of the emission from Ar II in a HCD. Spontaneous emission is the main loss mechanism and dominates over diffusion and electron superelastic de-excitation at our discharge conditions. The equilibrium density of upper levels of the Ar II transitions measured can be roughly described by:

$N_{i}^{+*}=\sigma_{i}^{+*}\left(J^{-} / e\right) N_{0} \tau_{+i}$,

where $N_{0}$ is the density of the neutral ground state, $\sigma^{+*}$ the cross section for one step electron excitation from the neutral ground state, $J^{-}$the discharge current density of beam electrons, $\tau_{+i}$ the lifetime of the $i$ th level of Ar II, and $e$ the electron charge.

The neutral argon levels excited in the HCD are also populated primarily in one step by beam electrons from

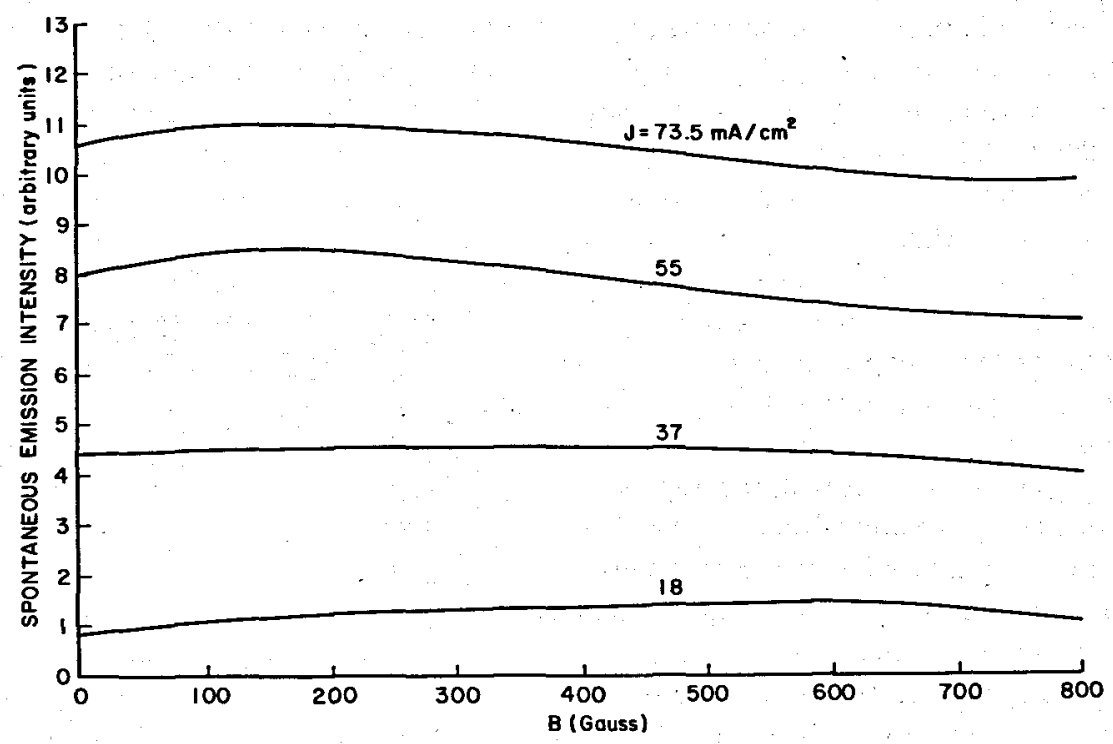

Fig. 2. Spontaneous emission intensity of Ar I 5916.6 \& line as a function of magnetic field strength. Filling pressure 2 Torr of argon. 


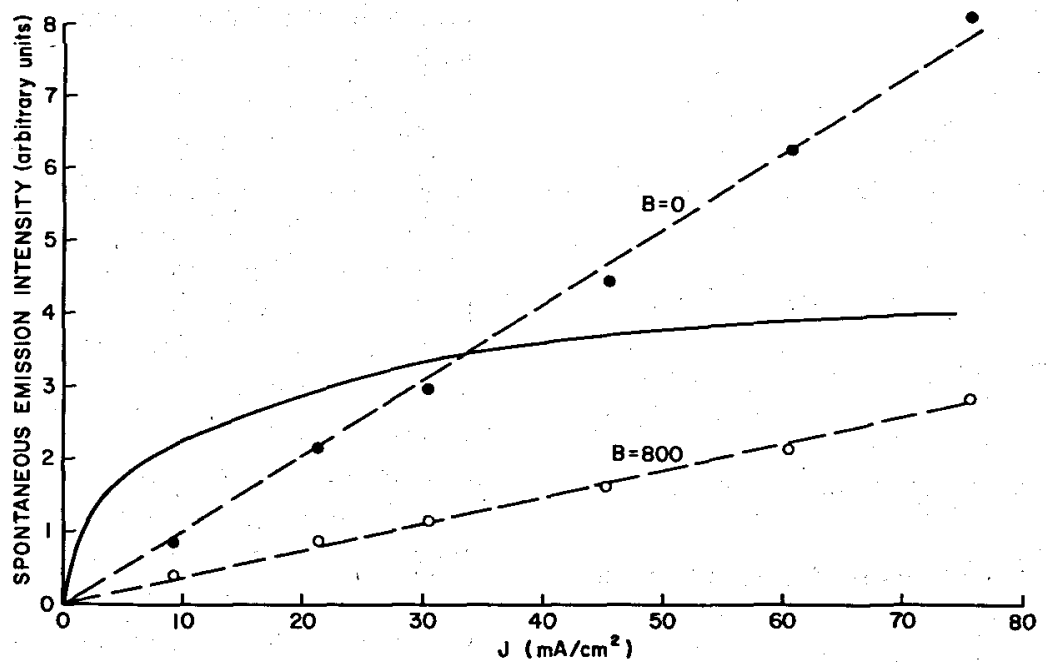

Fig. 3. Spontaneous emission intensity as a function of current. - - - Ar II 5143.3 \&, _ Ar I 4510.7 \&. Filling pressure 2 Torr of argon.

the atomic ground state, and depopulated by spontaneous emission and electron depopulation, mainly due to the large ionization rate of the excited levels of Ar I [8]. The equilibrium density $N_{i}^{*}$ of the Ar I levels then may be described by:

$N_{i}^{*}=\frac{\sigma_{i}^{*}(J / e) N_{0}}{B_{i} J^{-} / e+\tau_{i}^{-1}}$,

where $\sigma_{i}^{*}$ is the electron impact excitation cross section from the atom ground state, $B_{i}$ the "effective" electron de-excitation rate constant, and $\tau_{i}$ is the radiative lifetime of the level. For small currents eq. (2) shows a linear increase of the excited neutral population with current density, whereas at large current densities superelastic electron de-excitation and excitation to higher energy states become important and the population saturates, in agreement with the behavior of the Ar I spontaneous emission studied (fig. 3).

The observed behavior of the spontaneous emission intensity of the Ar I and Ar II lines with the applied magnetic field can also be explained by eqs. (1) and (2) via the magnetic field induced decrease of the effective current of beam electrons in the hollow cathode. Recall that the emission from the Ar II levels decreases significantly, while the emission from the nearly saturated atomic transitions does not change much. Moreover, the increase in magnetic field is also observed to cause the discharge voltage to decrease. This variation, more pronounced at low pressure (1.5 Torr), is shown in fig. 4. The behavior of the discharge voltage with the magnetic field is qualitatively similar to the variation of the spontaneous emission of the ion lines shown in fig. 1 .

Both the $q \mathbf{v} \times \boldsymbol{B}$ force, and the $\boldsymbol{E} \times \boldsymbol{B}$ drift force which exist in the dark space [9]; act together to trap the beam electrons in the cathode fall region and in the borders of the negative glow. These forces decrease the electron beam current density in the center of the negative glow and as a consequence when the magnetic field

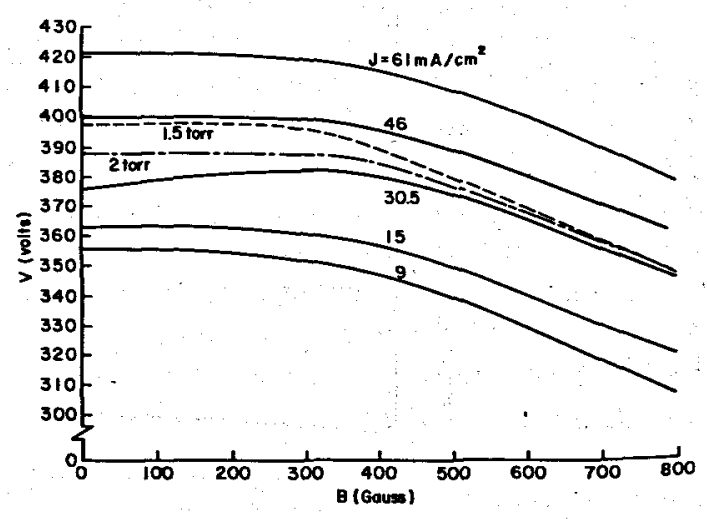

Fig. 4. Variation of the discharge voltage as a function of magnetic field strength. Filling pressure -2.5 Torr, - 2 Torr, - - 1.5 Torr of argon. 

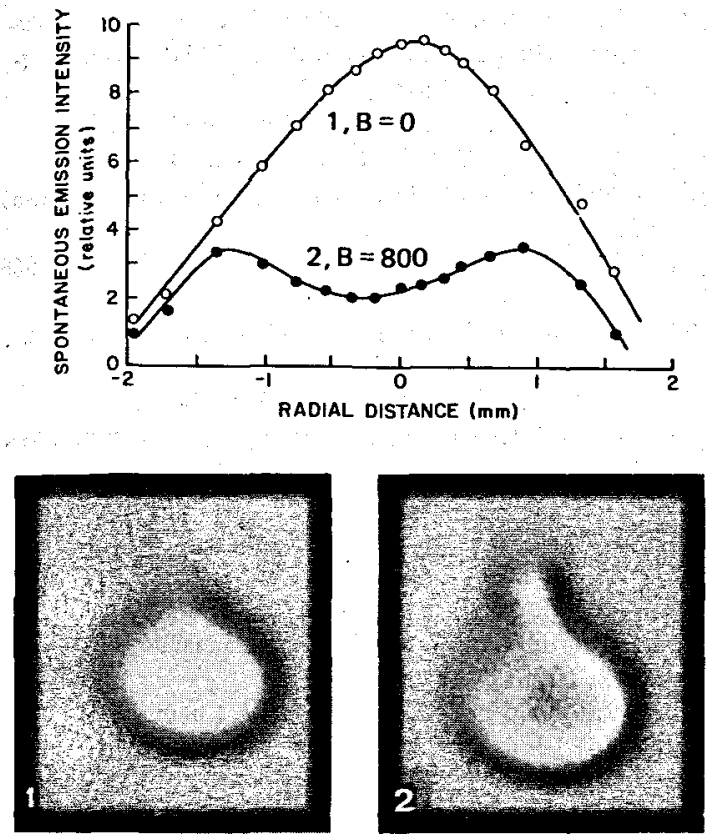

Fig. 5. Radial profile of Ar II $4764.8 \AA$ for magnetic field strengths of (1) $B=0 \mathrm{G}$ and (2) $B=800 \mathrm{G}$. Filling pressure 2.5 Torr of argon.

is applied externally a dark spot appears in the center of the discharge, as shown in fig. 5. Fig. 5 also shows spatially resolved measurements made of the spontaneous emission of an Ar II line. Notice that when the axial magnetic field is applied to the discharge, the emission is larger in the border of the discharge than in the axis. This is in contrast with the case of HCD operation at zero fields where the spontaneous emission peaks at the center of the cathode following the electron beam current density which also peaks on axis in a cylindrical HCD geometry. The electron trapping in the dark space reduces the positive space charge, $\rho$, in the cathode fall which in turn causes the observed reduction in discharge voltage (fig. 5), since according to ref. [10]:

$V_{c}=2 \pi \mathrm{d}^{2} \rho$

where $V_{\mathrm{c}}$ and $d$ are the dark-space voltage drop and dark-space thickness, respectively.

For all practical purposes the voltage drop in the cathode fall region ( $400 \mathrm{~V}$ ) can be considered to be equal to the HCD voltage. The voltage drop across the negative glow and the positive column region are small
$(<10 \mathrm{~V})$. Since in the absence of magnetic field electron collisions in the narrow dark space are negligible, the energy of the beam electrons is roughly equal to the applied voltage. Because our HC tube diameter is nearly one order of magnitude smaller than the reaching distance of $400 \mathrm{eV}$ electrons at our usual working pressure ( 2 Torr), an increase of the effective electron beam current $J_{\text {eff }}^{-}$occurs in the $\mathrm{HC}$ due to trapping of the electrons between the geometrically opposing cathode walls, and $J_{\text {eff }}^{-}$can be estimated to be

$J_{\text {eff }}^{-}=J \frac{\gamma}{1+\gamma} \frac{L}{d}$,

where $\gamma$ is the secondary electron emission coefficient, $d$ is the cathode diameter and $L$ is the reaching distance of the beam electrons with energy $e V_{c}$, as pointed out by McNeil [11]:

$L=\frac{1}{P V_{i}} \int_{V_{\mathrm{c}}}^{V_{i}} \frac{\mathrm{d} V}{\beta s(V)}$,

where $s(V)$ is the inverse ionization mean free path $\left(\mathrm{cm}^{-1}\right)$ in a gas at 1 Torr and $P$ the gas pressure, $e V_{i}$ is the ionization energy and $\beta$ is a factor which accounts for the fact that more energy than $e V_{i}$ is expended by a beam electron in each ionization process. We notice that the reduction of the discharge voltage by the applied magnetic field reduces the effective electron beam current by reducing the $\mathrm{HC}$ trapping factor $L / d$. For a pressure of 2.5 Torr and a current density of $60 \mathrm{~mA} / \mathrm{cm}^{2}$, we measured that a magnetic field of $800 \mathrm{G}$ causes a reduction of $10 \%$ in the discharge voltage. Taking $\beta=1.7$ [12] and using ionization data from ref. [13] from formulas (4) and (5) we conclude this reduces the effective electron beam current by $15 \%$. According to expression (1) this effective current drop due to a discharge voltage decrease accounts for $30 \%$ of the total observed reduction in the intensity of the ion lines. The rest is attributed to the fact that the electrons arrive at the negative glow with an energy less than $e V_{c}$, since they are likely to loose energy colliding in the cathode fall region when the magnetic field partially traps them there. The observed decrease in discharge voltage confirms the fact that the electrons are partially trapped in the dark space; however, the two phenomena of charge trapping and energy loss cannot be separated. The percentages attributed to each phenomenon are only given to show that both effects have to be considered to fully explain 
the observed reduction in the intensity of the spontaneous emission of the ion lines.

In summary, an axial magnetic field in an argon hollow cathode discharge decreases the population of $\mathrm{Ar}$ II levels, but the population of Ar I excited levels, that are observed to nearly saturate at low current densities, is not significantly altered. Since the magnetic field causes a reduction of the effective electron beam current in the negative glow, its use in an argon ion hollow cathode laser would only reduce the laser output in ways similar to those reported in $\mathrm{He}-\mathrm{Cd}$ hollow eathode lasers [7].

\section{References}

[1] D.C. Gerstenberger, R. Solanki and G.J. Collins, IEEE J. Quantum Electron. QE-16 (1980) 820.
[2] W.K. Schuebel, in: Proc. Intern. Conf. on Ionized phenomena (Prague, Czechoslovakia, 1973) (Czech Academy of Science, Prague, 1973).

[3] I. Gorog and F. Sprong, RCA Rev. 26 (1965) 38.

[4] C.P. Cristescu, Can. J. Phys. 56 (1978) 1071.

[5] M. Somesan and C. Popovici, Appl. Phys. Lett. 9 (1966) 65.

[6] C. Popovici and M. Somesan, Appl. Phys. Lett. 8 (1966) $5,103$.

[7] C.P. Cristescu, I.M. Popescu and A.M. Preda, J. Phys. D12, (1979) 845.

[8] C.E. Webb, J. Appl. Phys. 39 (1968) 5441.

[9] F. Chen, Introduction to plasma physics (Plenum, New York, 1977) p. 21.

[10] J. Cobine, Gaseous conductors (Dover Publ., New York, 1958) p. 221.

[11] J. R. McNeil, New sputtered metal vapor laser systems, Dissertation, Colorado State Univ. (1977).

[12] G. Bakafi, Principles of laser plasmas (Wiley, New York, 1976) p. 373.

[13] D. Rapp and P. England-Golden, J. Chem. Phys. 43 (1965) 1464. 